\title{
A rare case of tuberculoma masquerading as CP Angle neoplasm
}

\author{
Sinjan Ghosh ${ }^{1}$, Gautam Guha², Kaushik Roy ${ }^{3}$, Annesh Bhattacharjee ${ }^{4}$, Nikhil Repaka ${ }^{5}$, \\ Sourav Nanda ${ }^{6}$, Niraja Agasti \\ 1,4,5,6,7 Neurology Resident, ${ }^{2}$ Associate Professor, Department of Neurology, Nil Ratan Sircar Medical College and \\ Hospital, Kolkata, West Bengal, India, ${ }^{3}$ Associate Professor, Department of Neurosurgery, Nil Ratan Sircar Medical \\ College and Hospital, Kolkata
}

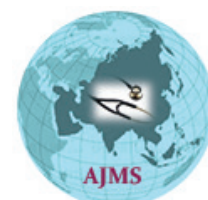

A B S T R A C T

Tuberculoma involving the cerebellopontine angle is very rare. Preoperative neuroradiological features of such lesions may mimic neoplastic lesions. Our case presented with cerebellar features and multiple cranial nerve palsy. Neuroimaging mimicked CP angle neoplastic lesion. Antitubercular therapy and steroids resulted in significant clinical improvement and marked radiological reduction in size of the lesion. In our subcontinent a treatable infective cause like tuberculosis should be ruled out in $\mathrm{CP}$ angle lesions. Although rare but definitely a possibility to be considered.

Key words: CP Angle SOL; Tuberculoma; CNS Tuberculosis; Tuberculosis radiology

\section{Access this article online}

Website:

http://nepjol.info/index.php/AJMS DOI: 10.3126/ajms.v10i4.24169

E-ISSN: 2091-0576

P-ISSN: 2467-9100

\section{INTRODUCTION}

Approximately $10-15 \%$ of all patients with tuberculosis have central nervous system (CNS)involvement ${ }^{1}$ and it is seen most commonly in the developing countries. Although large series from developing countries continue to be reported ${ }^{2}$ and incidence has increased for the last 20 years due to human immunodeficiency virus (HIV) infection, people of any age can be affected and the mycobacterium usually reaches the CNS by haematogenous dissemination from pulmonary lesions.

Tuberculoma is found in $15-30 \%$ of cases of CNS tuberculosis ${ }^{3}$ and is hemispheric in most cases ${ }^{2}$. Other, rare locations include the sellar area, cerebellopontine angle, Meckel's cave, suprasellar cistern, and hypothalamic region ${ }^{3,4}$

Central nervous system tuberculoma presenting as a solitary mass is rare in an extrinsic location. The cerebellopontine angle is a very unusual site for tuberculoma ${ }^{5}$. Only a few reports have been published in the literature.

\section{CASE REPORT}

A 47 yrs old normotensive and euglycemic right handed gentleman presented with a subacute onsetprogressively increasing left hemicranial headache with low grade intermittent fever for 4 months.

The headache was dull aching and occasionally throbbing in character and of moderate to severe in intensity. Within 3 weeks he developed facial deviation towards the right side with diminished hearing in his left ear with speech discrimination more affected than pure tones. Within 1 week this was followed by tremulousness of limbs and gait imbalance with tendency to fall towards the left side.

There was a background history of tremulousness of head and limbs for long 20yrs with similar history in his father. There was no history suggestive of cognitive affection, sphincter complains, bulbar or sensory involvement.

On Clinical examination, patient was conscious oriented with dysarthric speech. Saccades slow with broken pursuits and gaze evoked nystagmus with fast component to the left side. Left LMN facialpalsy and mixed (Conductive and Sensorineural) deafness in left ear. Features consistent with left sided vestibular affection was also noted. Motor power was $4 / 5$ in right upper and lower limbs with normal power in left side. Postural tremor was noted in bilateral upper limbs(right more than left)with dystonic posturing and in left upper limb intention tremor was present. Head 
tremor was also noted. Sensory system examination was normal. Deep tendon reflexes were preserved with left plantar flexor and right plantar was equivocal. Left sided cerebellar signs were elicited. He had a wide based lurching and ataxic gait. Meningeal signs were absent. Cranium and spine examination was within normal limits.

On routine lab investigations ESR was $82 \mathrm{~mm}$ in $1^{\text {st }} \mathrm{hr}$ and Mantoux test was positive. Other investigations including Chest $\mathrm{X}$ ray were normal.

MRI Brain (Plain and Contrast) [08/10/2018] showed extra axial SOL in Left CP Angle with intracanalicular extension along with meningeal enhancement and perilesional edema (Figure 1 and 2). Although the neuroimaging was reminiscent of a CP angle neoplastic SOL, the clinical profile and minute observation of the lesion evoked a suspicion of infective pathology consistent with a chronic granulomatous inflammation. A tissue diagnosis was planned but the patient and his caregivers refused to go for surgical exploration considering the probable inadvertent consequences.

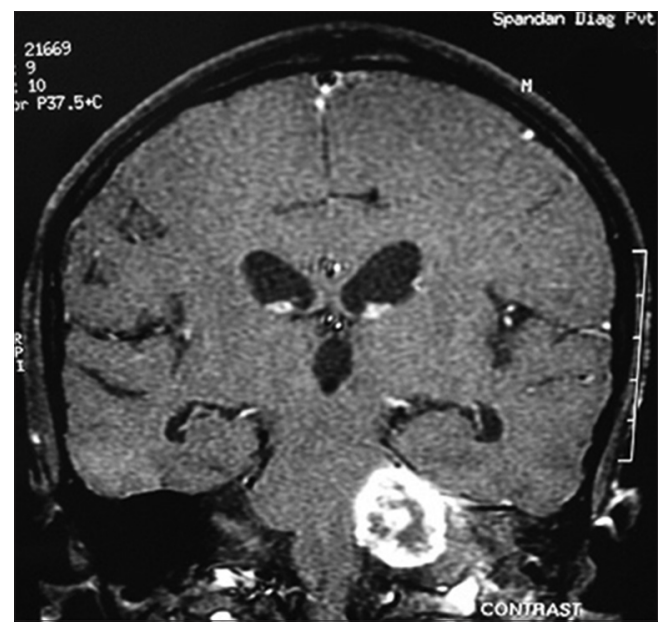

Figure 1: Left CP angle SOL- T1 post contrast image

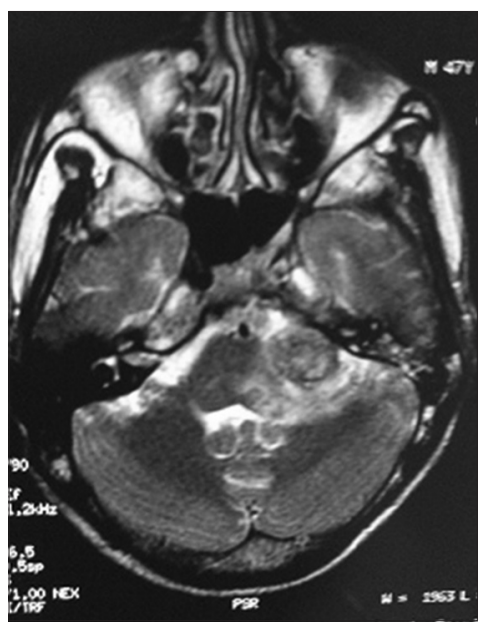

Figure 2: Left $\mathrm{CP}$ angle $\mathrm{SOL}$ with edema in $\mathrm{T} 2 \mathrm{~W}$ axial image
On high clinical suspicion anti tubercular therapy with steroid was administered as per protocol and MR Spectroscopy of the lesion was ordered. MR Spectroscopy and high (Figure 3a and 3b) ESR was supportive of a Tubercular etiology in this case. In the course of treatment his headache and other symptoms subsided.

A repeat MRI Brain (Plain and Contrast) after 1 month of ATD revealed- The lesion in left CP Angle got reduced in size with resolving perilesional edema as compared to the previous MRI Figure 4.

This report was corroborating with the clinical improvement. He was discharged with mild head and upper limb tremor which was present since long and was familial. A residual left LMN Facial palsy and mixed deafness in left ear persisted. He is on follow up and doing well clinically.

\section{DISCUSSION}

With the increasing number of tuberculosis cases, CNS tuberculosis is also increasing with 3-5\% casespresenting with isolated CNS affection without pulmonary involvement. ${ }^{5}$

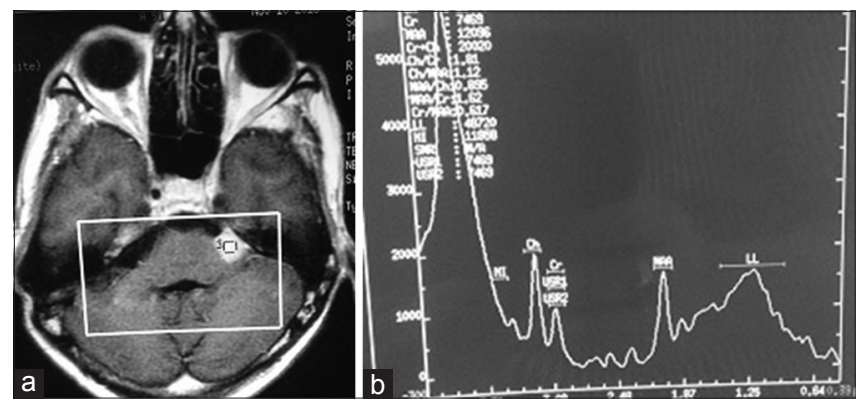

Figure 3: (a) MRS voxel on CP angle SOL (b) MRS on lesion showing lipid lactate peak

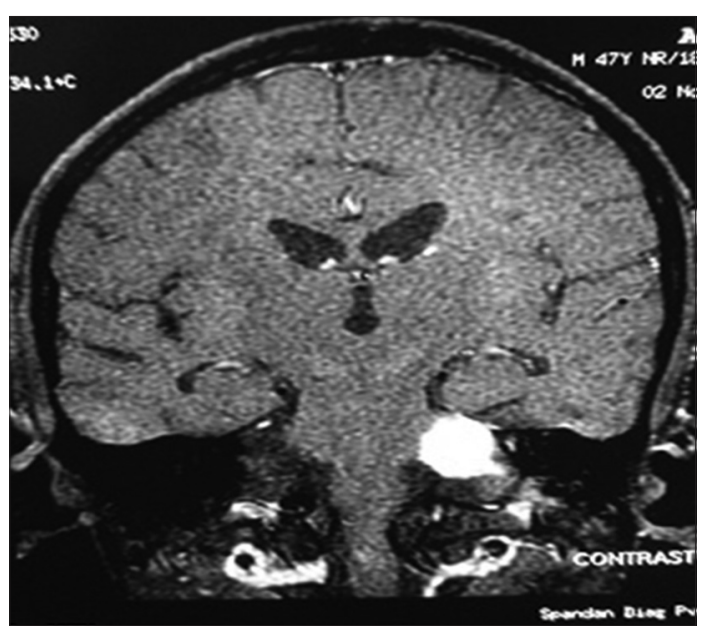

Figure 4: Post ATD treatment reduction in size of SOL-post contrast

Asian Journal of Medical Sciences | Jul-Aug 2019 | Vol 10 | Issue 4 
Tuberculosis involving the leptomeninges, brain and skull base is thought to spread by haematogenous dissemination from a primary source outside the CNS. The meningitic process may affect the cranial cerebrospinal fluid (CSF) pathway, the spinal subarachnoid pathway, or both ${ }^{6}$ Parenchymal disease usually presents as either solitary or multiple tuberculoma, and can occur with or without meningitis? ${ }^{7}$ Tuberculomas are histologically round or oval masses, or they may assume a more lobular configuration with the fusion of several smaller nodules. ${ }^{3}$

In the present case the classical features were lacking.

Only ten percent of CNS TBare associated with concomitant meningitis ${ }^{8}$. We did not perform a CSF study, but there were no clinical evidence of meningitis or intraventricular lesions apart from a small lesion in left paraventricular region suggestive of an isolated tuberculoma. On meticulous observation the CP angle SOL showed multiple conglomerated ring enhancing lesions with perilesional edema.

Confirmation of CNS tuberculosis is only possible by isolation of Mycobacterium or histopathological identification of tuberculous granuloma. In all cases tissue diagnosis is not possible and ATD is initiated on the basis of strong clinical suspicion. Various investigations including CTscan, MRI, magnetization transfer MRI, or in vivo proton MR-spectroscopy ${ }^{9}$ have been suggested.

The inappropriate neuroradiological diagnosis of meningioma, neurinoma, or even metastasis is usual in cases of tuberculoma ${ }^{10}$. Cerebellopontine angle tumours are most common lyacoustic schwannomas or meningiomas. ${ }^{5}$ Other differential diagnoses include cholesteatoma,metastases, lymphoma, brain abscess, fungal infection, neurocysticercosis, sarcoidosis, etc. Tuberculomas occurring as isolated masses in thecerebellopontine angle are extremely rare, with onlytwo cases reported, both of which were in the pre- MRI era ${ }^{5}$ followed by a very few cases reported after that.

\section{CONCLUSION}

The uniqueness of our case is the rare presentation of a treatable infective pathology radiologically masquerading as a neoplastic SOL. The histopathological evidence could not be confirmed as the patient refused any surgical intervention but the clinical pointers and epidemiological probability being high, ATD was administered. The MR Spectroscopy was supportive and the therapeutic response was the clincher in this case.

\section{REFERENCES}

1. Prabhakar S., Thussu A. Central nervous system tuberculosis. Neurol India 1997; 45: 132-140.

2. Arvind C., \& Korath M.P., Raveendranadhan, K \& Jagadeesan, K. A retrospective study of 1247 cases of intracranial tuberculomata diagnosed by computerized tomography. J Assoc Physicians India 1993; 41: 559-61.

3. de Castro C.C., de Barros N.G., Campos Z.M., Cerri GG. CT scans of cranial tuberculosis. RadiolClin North Am 1995;33: 753-769.

4. Goel A., Nadkarni T., Desai A.P. Tuberculoma in the Meckel'scave: a case report. Neurol India 1999; 47: 238-240.

5. Sathyanarayana S., Baskaya M.K., Fowler M., Roberts R., Nanda A. Solitary tuberculoma of the cerebellopontine angle: a rare presentation.J ClinNeurosci 2003; 10: 120-122.

6. Khoo J.L.S., Lau K.Y., Cheung C.M., Tso T. H. Central nervous system tuberculosis. J HK CollRadiol 2003; 6: 217-228.

7. Jinkins J.R. Computed tomography of intracranial tuberculosis. Neuroradiology 1991; 33: 126-135.

8. Giese A., Kucinski T., \& Hagel C., \& Lohmann F. Intracranial tuberculomas mimicking a malignant disease in an immunocompetent patient. Acta Neurochir 2003. 145. 513-7; discussion 517.

9. Gupta R.K., Husain M., Atsal D.K., Kumar R., Chawla S., Husain N. Comparative evaluation of magnetization transfer MR imaging and in vivo MR spectroscopy in brain tuberculomas. Magn Reson Imaging 2002; 20: 375-381.

10. Yanardag H., Uygun S., Yumuk V., Caner M., Canbaz B. Cerebral tuberculosis mimicking intracranial tumour. Singapore Med J 2005; 46: 731.

\footnotetext{
Authors Contribution:

SG and KR: Concept and design of the study, reviewed the literature, manuscript preparation and critical revision of the manuscript; GG: Concept, collected data and review of literature and helped in preparing first draft of manuscript; AB and NR: Conceptualized study, literature search, statistically analyzed and interpreted, prepared first draft of manuscript; SN and NA: Concept of study, collected data and review of study.

Work attributed to:

Department of Neurology, Nil Ratan Sircar Medical College and Hospital, Kolkata, West Bengal, India

Orcid ID:

Dr. Sinjan Ghosh- 1 https://orcid.org/0000-0003-2416-3181

Dr. Gautam Guha- (I) https://orcid.org/0000-0002-8902-2817

Dr. Kaushik Roy- it htps://orcid.org/0000-0001-8317-9840

Dr. Annesh Bhattacharjee- (1) http://orcid.org/0000-0001-6560-2692

Dr. Nikhil Repaka- http://orcid.org/0000-0002-3856-8940

Dr. Sourav Nanda- http://orcid.org/0000-0003-2049-8208

Dr. Niraja Agasti- isttp://orcid.org/0000-0001-5884-719X

Source of Support: Government of West Bengal, India, Conflict of Interest: None declared.
} 